\title{
The RNA-binding landscapes of two SR proteins reveal unique functions and binding to diverse RNA classes
}

\author{
Minna-Liisa Änkö ${ }^{1,4^{*}}$, Michaela Müller-McNicoll1 ${ }^{1}$, Holger Brandl ${ }^{1}$, Tomaz Curk ${ }^{3}$ Crtomir Gorup ${ }^{3}$, lan Henry', \\ Jernej Ule ${ }^{2}$ and Karla M Neugebauer ${ }^{1 *}$
}

\begin{abstract}
Background: The SR proteins comprise a family of essential, structurally related RNA binding proteins. The complexity of their RNA targets and specificity of RNA recognition in vivo is not well understood. Here we use iCLIP to globally analyze and compare the RNA binding properties of two SR proteins, SRSF3 and SRSF4, in murine cells.

Results: SRSF3 and SRSF4 binding sites mapped to largely non-overlapping target genes, and in vivo consensus binding motifs were distinct. Interactions with intronless and intron-containing mRNAs as well as non-coding RNAs were detected. Surprisingly, both SR proteins bound to the $3^{\prime}$ ends of the majority of intronless histone transcripts, implicating SRSF3 and SRSF4 in histone mRNA metabolism. In contrast, SRSF3 but not SRSF4 specifically bound transcripts encoding numerous RNA binding proteins. Remarkably, SRSF3 was shown to modulate alternative splicing of its own as well as three other transcripts encoding SR proteins. These SRSF3-mediated splicing events led to downregulation of heterologous SR proteins via nonsense-mediated decay.

Conclusions: SRSF3 and SRSF4 display unique RNA binding properties underlying diverse cellular regulatory mechanisms, with shared as well as unique coding and non-coding targets. Importantly, CLIP analysis led to the discovery that SRSF3 cross-regulates the expression of other SR protein family members.
\end{abstract}

\section{Background}

Gene expression in metazoans is regulated at multiple levels. While investigation of transcriptional regulation by transcription factors has led to a deep understanding of how DNA binding proteins direct RNA polymerases to genes, regulation of RNA processing by RNA-binding proteins is still enigmatic. Hundreds of proteins encoded by metazoan genomes have RNA-binding capacity conferred by specific protein structural domains, such as RNA recognition motifs (RRMs), KH domains and zinc fingers [1]. RNA-binding proteins can change gene expression output at different steps of RNA metabolism, including pre-mRNA splicing, polyadenylation, RNA export, RNA stability, and translation. However, the in

\footnotetext{
* Correspondence: minna-liisa.anko@monash.edu; neugebau@mpi-cbg.de ${ }^{1}$ Max Planck Institute of Cell Biology and Genetics, Pfotenhauerstr. 108, Dresden, 01307, Germany

Full list of author information is available at the end of the article
}

vivo binding specificity and function(s) of most RNAbinding proteins are not well understood.

SR proteins are a family of seven RNA-binding proteins with a functional repertoire that has expanded to many aspects of RNA metabolism [2,3]. They are concentrated in the nucleus, where they participate in premRNA splicing [4], yet nearly all SR proteins shuttle between the nucleus and cytoplasm. SR protein shuttling activity contributes to their roles in mRNA export, stability and translation $[5,6]$. SR proteins share a modular structure of one or two RNA recognition motifs (RRMs) at their amino terminus and an arginine-serine-rich RS domain of variable length at the carboxyl terminus. Both domains can directly contact RNA [7], although the RRM appears to determine RNA-binding specificity $[5,8,9]$. In vitro binding specificities have been determined for some SR protein family members $[10,11]$, which bind to 4- to 10-nucleotide long degenerate sequences. Recently, in vivo crosslinking was used to
C Biomed Central

ㄷ 2012 Änkö et al.; licensee BioMed Central Ltd. This is an open access article distributed under the terms of the Creative Commons Attribution License (http://creativecommons.org/licenses/by/2.0), which permits unrestricted use, distribution, and reproduction in any medium, provided the original work is properly cited. 
define mRNA targets of SRSF1 (also called ASF/SF2); this study identified thousands of SRSF1 target sites, which resembled the sequences derived in vitro [12]. Mature mRNAs associated with SRSF3 (SRp20) and SRSF4 (SRp75) were also recently identified and represent functionally distinct mitochondrial ribonucleoproteins (mRNPs) [13]. However, the latter analysis provided information at the gene level and did not identify direct binding sites of SR proteins to RNA targets.

To understand the widespread functions of SR protein family members, the identification of endogenous RNA target sites is required. The development of ultraviolet (UV) crosslinking and immunoprecipitation (CLIP) followed by high-throughput sequencing has made possible the identification of in vivo binding sites of RNA-binding proteins in a genome-wide manner [14]. Here we used a modification of the CLIP protocol called iCLIP [15], which allows high-resolution identification of RNA-protein crosslink sites, to investigate the binding specificity and endogenous RNA targets of SRSF3 and SRSF4. We took advantage of our previously developed tagging and stable expression system, in which an enhanced green fluorescent protein (EGFP) tag is inserted at the carboxyl terminus of the SR protein by recombineering of bacterial artificial chromosomes (BACs); due to co-regulation of the endogenous and stably integrated transgenes, the total level of SR protein expression is unchanged in the diploid mouse P19 cells used here [13]. Using the EGFP tag as a universal epitope for iCLIP, we determined in vivo binding sites of SRSF3 and SRSF4. Our analysis shows that SRSF3 and SRSF4 bind to distinct sequences and target RNAs, including non-coding RNAs (ncRNAs). The subsequent analysis showed that SRSF3 or SRSF4 binding to these sites conferred regulatory functions in several steps of RNA metabolism in cells, supporting the widespread contribution of SR proteins in gene expression regulation.

\section{Results}

\section{SRSF3 and SRSF4 bind distinct RNAs}

We used the iCLIP method [15] to identify SRSF3 and SRSF4 binding sites genome-wide in mouse P19 cells. SRSF3 and SRSF4 were immunopurified via the EGFP tag encoded on stable transgenes to allow direct comparison of the binding profiles of the two SR proteins [13]. Previous analyses showed that the EGFP-tagged SR proteins recapitulate interactions with nascent RNA and functionally rescue the endogenous proteins $[5,13]$. Both SRSF3-EGFP and SRSF4-EGFP were specifically and efficiently immunopurified from cell extracts, and SR protein-RNA complexes were isolated after in vivo UV crosslinking (Figure S1a, b in Additional file 1). No RNA-protein complexes were detected in cells expressing only nuclear EGFP (EGFP-nuclear localization signal) or in the absence of UV crosslinking (Figure S1b in Additional file 1). In each replicate experiment, SRSF4 showed weaker signal intensity than SRSF3 (Figure S1b in Additional file 1), indicating either lower crosslinking efficiency or fewer RNA targets.

Crosslinked, immunopurified RNA was digested to lengths of 40 to 100 nucleotides, reverse transcribed and prepared for next-generation sequencing [15] (Figure S1c in Additional file 1). The resulting reads, referred to as CLIP-tags throughout the manuscript, were aligned to the mouse $\mathrm{mm} 9$ genome assembly. In total, iCLIP produced 1,212,480 and 243,501 unique CLIP-tags for SRSF3 and SRSF4, respectively (Table S1 in Additional file 1). SRSF4 reproducibly yielded fewer sequence reads, in agreement with the lower crosslinking levels observed (Figure S1b in Additional file 1). The EGFP-nuclear localization signal control iCLIP experiments performed in parallel did not produce any detectable PCR products and yielded a total of 2,611 CLIP-tags mapping to the mouse genome. Because the SRSF3 and SRSF4 iCLIPs generated 100 - to 1,000 -fold more CLIP-tags than the control iCLIP, less than $1 \%$ of the detected CLIP-tags could be due to nonspecific crosslinking.

As a first step towards analyzing the RNAs and RNA regions bound by SRSF3 and SRSF4, crosslink sites were identified by mapping to the first nucleotide upstream of the start of each CLIP-tag, as previously described [15]. We determined statistically significant SRSF3 and SRSF4 crosslink sites (33,458 and 10,393, respectively), and identified CLIP-tag clusters with a maximum spacing of 15 nucleotides and containing a significant CLIP-tag count when compared to randomized positions (false discovery rate $<0.05$ ) [15-17]. To test whether the iCLIP captured only the most highly expressed genes, we compared the density of CLIP-tags to our global gene expression data in P19 cells [13]. There was a slight positive correlation between the gene expression level and the density of CLIP-tags within the gene, yet CLIP-tags were identified in genes at the whole range of gene expression (Figure S1d in Additional file 1).

Examination of SRSF3 and SRSF4 CLIP-tag clusters indicated that multiple reads were detected in limited RNA regions. The same transcript could display crosslinking to both SR proteins, albeit in different regions of the transcript, as exemplified by the NPM1 gene that contained CLIP-tag clusters for both SRSF3 and SRSF4 mapping to distinct exons (Figure 1a). Also at the chromosome level, a large proportion of the CLIP-tags and clusters were non-overlapping (Figure 1a; Figure S2 in Additional file 1). Significant crosslink sites were detected in 2,304 genes for SRSF3 and 1,055 genes for SRSF4, of which $83.3 \%$ and $83.2 \%$ were protein-coding, 


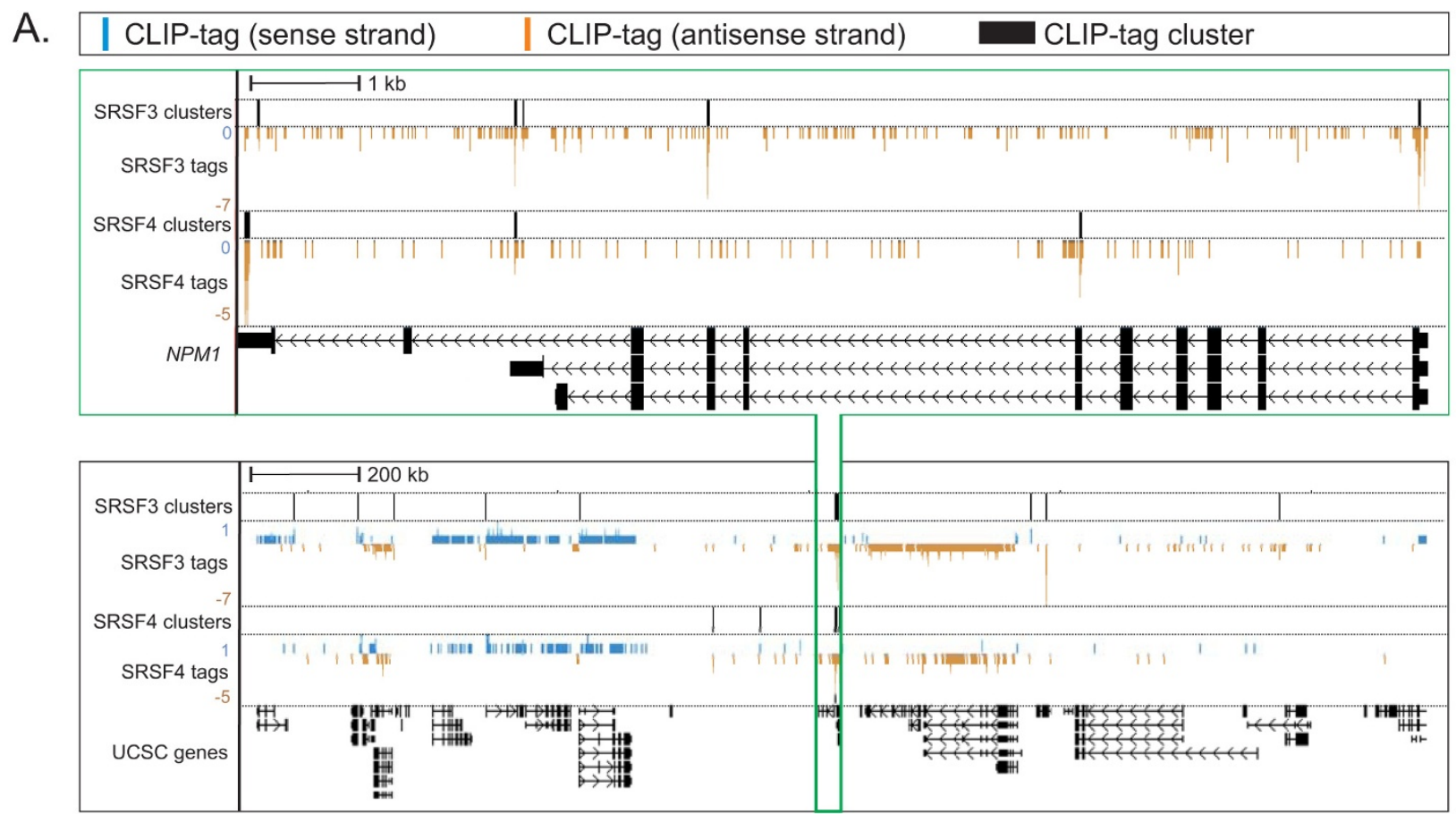

B. Genes with significant crosslink sites

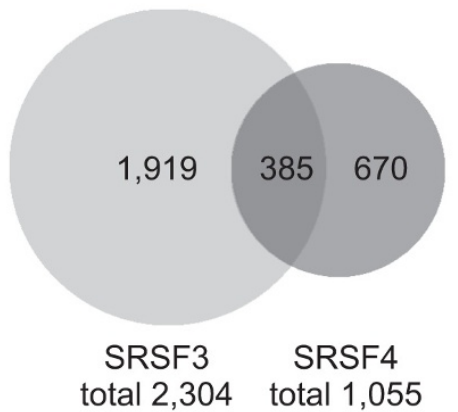

C. Significant CLIP-tag clusters

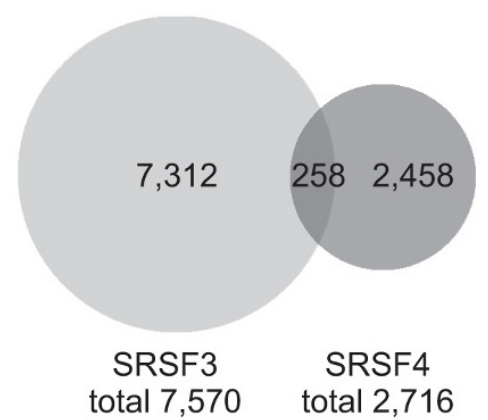

Figure 1 SRSF3 and SRSF4 CLIP-tags cluster to distinct positions in mouse RNAs. (a) NPM1 gene (green box) and the surrounding approximately 3 MB region in chromosome 11 (black box) with SRSF3 and SRSF4 CLIP-tags and clusters. The numbers on the left represent the number of CLIP-tags within the window. The sense strand is marked in blue and the antisense strand in orange. Note that the genes in the antisense strand run from right to left. (b) Comparison of annotated genes with significant SRSF3 or SRSF4 crosslink sites (false discovery rate < 0.05). (c) Comparison of significant SRSF3 and SRSF4 CLIP-tag clusters (overlap of clusters $\geq 15$ nucleotides).

respectively. A list of genes with significant crosslink sites is provided in Additional file 2. These numbers are likely to be underestimates because our sequencing has not reached saturation. In agreement with our recent analysis showing that SRSF3 and SRSF4 associate with distinct mRNAs [13], the identity of the target RNAs bound by SRSF3 and SRSF4 only partially overlapped (Figure 1b). An even smaller overlap between SRSF3 and SRSF4 CLIP-tag clusters, rather than genes, was observed (compare Figure $1 \mathrm{~b}$ and 1c), strongly suggesting differential RNA-binding specificities of SRSF3 and SRSF4.

\section{Consensus binding motif of SRSF3 and SRSF4}

The in vivo binding specificities of SRSF3 and SRSF4 are unknown. The differences in the CLIP-tag cluster sites suggested that each of the two SR proteins binds to a distinct RNA sequence. To address this directly, we used the data to derive in vivo binding motifs for SRSF3 and SRSF4 by analyzing enriched pentamer sequences 
around the crosslink sites. To calculate a Z-score for each pentamer, iCLIP positions were randomized within the same regions. The pentamer enrichment analysis showed that SRSF3 and SRSF4 identify distinct sequence motifs (Figure 2). The top five pentamers for SRSF3 (Figure 2a) were in close agreement with the core SELEX (systemic evolution of ligands by exponential enrichment) motif determined in vitro $[18,19]$. SELEX has not been performed on SRSF4; interestingly, the SRSF4 top five pentamers (Figure 2b) were similar to one sequence (GAAGGA) previously shown to be an SRSF4 binding site in bovine papilloma virus pre-mRNA [20]. The SRSF3 binding motif was CU-rich excluding Gs, whereas SRSF4 bound to GA-rich sequences excluding Cs (Figure 2d). These results are consistent with the largely non-overlapping SRSF3 and SRSF4 crosslink sites and clusters (Figure 2c, and see above).

\section{SRSF3 and SRSF4 bind to coding and non-coding RNAs}

Which categories of RNA and which functional RNA regions are bound by SR proteins? Analysis of the frequency with which SRSF3 and SRSF4 CLIP-tags were mapped to genes and gene regions revealed their common propensity to bind exons and introns in proteincoding genes (Figure 3a; Table S3 in Additional file 1). The high proportion of intronic CLIP-tags detected clearly reflects the fact that mammalian introns are much longer than exons; when the frequency of CLIP-
A.

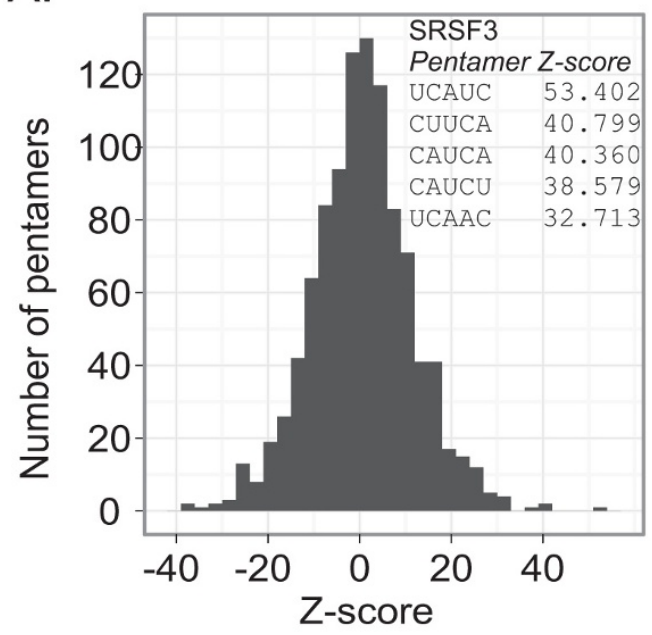

C.

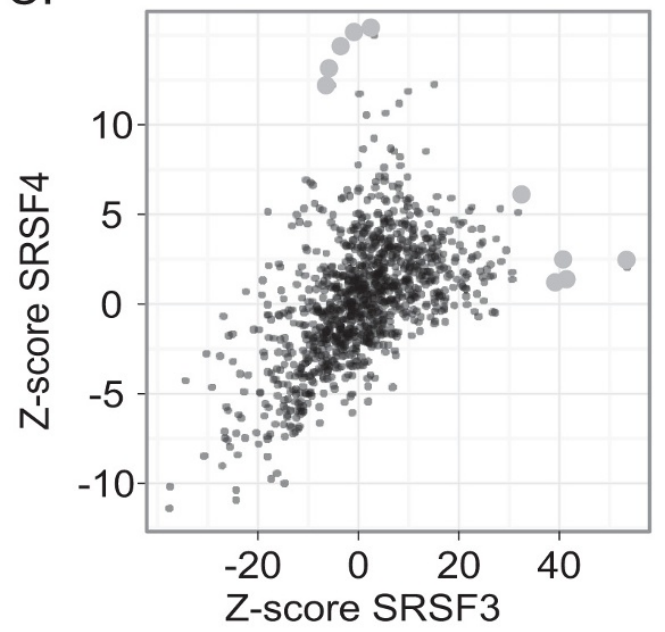

B.

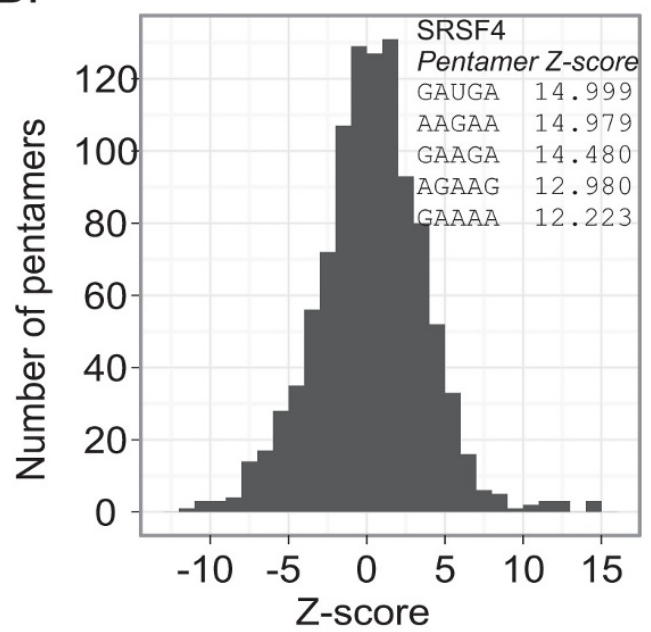

D.
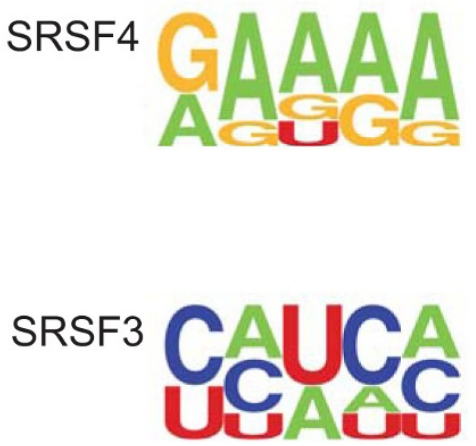

Figure 2 In vivo binding specificity of SRSF3 and SRSF4. (a, b) The frequency distribution of SRSF3 (a) and SRSF4 (b) pentamer Z-SCores. The $Z$-score was calculated relative to randomized genomic positions by shuffling the crosslink positions 100 times within the genes. Five pentamers with highest Z-scores are shown. (c) Correlation of SRSF3 and SRSF4 pentamer Z-scores. The top five pentamers presented in (a, b) are marked as larger light grey dots. (d) Consensus motifs were derived from the top pentamers shown in (a, b). 

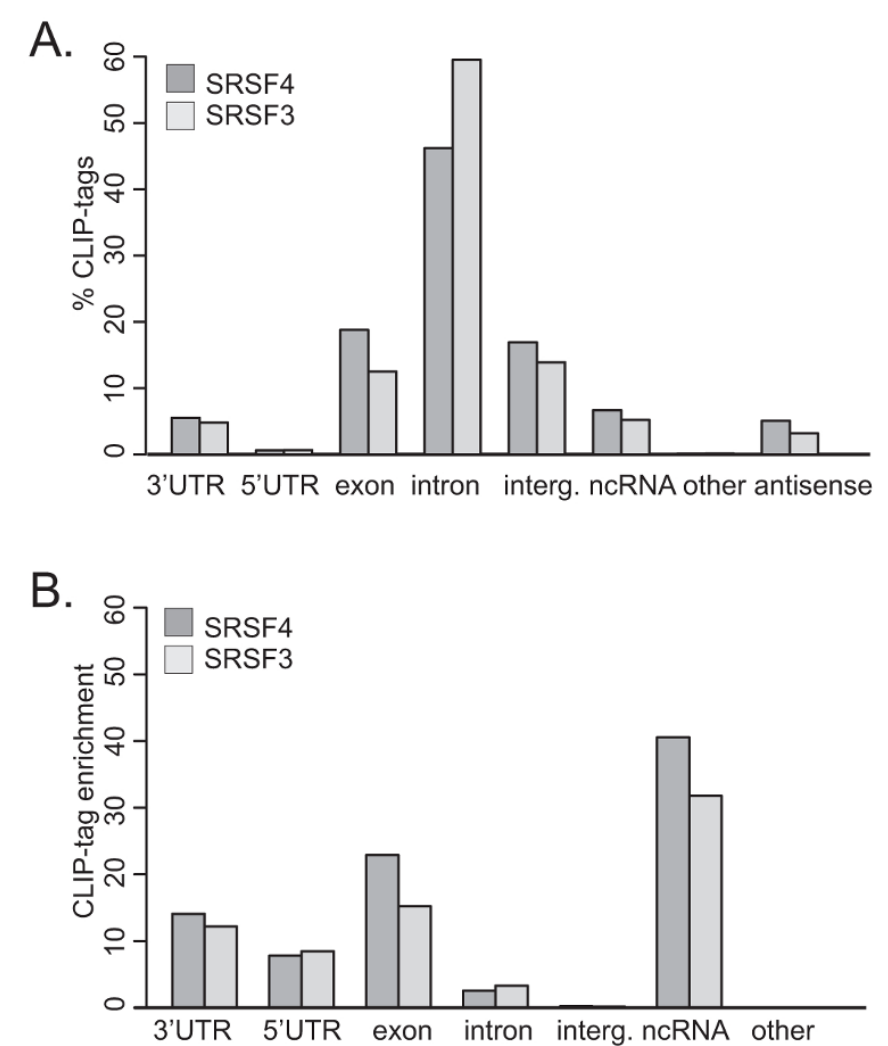

Figure 3 Distribution of SRSF3 and SRSF4 CLIP-tags within RNA classes and transcript regions. (a) The proportion of CLIP-tags that mapped to different RNAs relative to the total number of CLIP-tags. (b) The fold enrichment of CLIP-tag density (the number of CLIP-tags divided by the length of each RNA feature) in different RNAs relative to the average CLIP-tag density in the genome.

tags was normalized to the length of the RNA region (Figure 3b), both SRSF3 and SRSF4 CLIP-tags were more highly enriched in exons than in introns. SR protein interactions with exons could reflect activities either in pre-mRNA splicing or in mRNPs after splicing (see below).

The highest density of CLIP-tags was detected in ncRNAs (Figure 3b). Overall, 319 and 141 ncRNAs had SRSF3 and SRSF4 CLIP-tag clusters, respectively. The most abundant ncRNA classes with CLIP-tags were long ncRNAs (lincRNAs) and small nucleolar RNAs (snoRNAs) (Figure 4a). Similar to SRSF1 and TDP-43 [12,21], SRSF3 and SRSF4 crosslinked to the lincRNA MALAT1 (aka NEAT2; Figure S3a in Additional file 1) that is enriched in nuclear speckles [22]. In addition, another speckle-localized ncRNA, 7SK [23], had abundant SRSF3 and SRSF4 CLIP-tag clusters (data not shown). An especially large proportion of ncRNAs with SRSF3 and SRSF4 crosslink sites belonged to snoRNAs, a class of small RNAs that guide RNA modifying enzymes [24]. Intriguingly, small Cajal body-specific RNAs (scaRNAs), a subclass of snoRNAs, were enriched in SRSF4 CLIP-tag clusters.
SR protein binding could not be correlated with known elements within scaRNAs because the scaRNAs identified included those with H/ACA boxes alone, $\mathrm{C} /$ D boxes alone, and a combination of H/ACA and C/D boxes. The specificity of SR protein binding to this group of scaRNAs was investigated in two ways. First, we asked whether binding was biased to any particular region of the scaRNAs. Figure $4 \mathrm{~b}$ shows that binding sites were localized near scaRNA 3' ends (Figure 4b; Figure S3a in Additional file 1). Second, the CLIP-tag clusters within the scaRNAs were used to determine a consensus binding motif independent of the global pentamer analysis. Multiple alignment of the CLIP-tag cluster regions using the MEME (Multiple Em for Motif Elicitation) algorithm identified a consensus sequence element (Figure S3c in Additional file 1) that was found in all scaRNAs with SRSF4 CLIP-tag clusters. The motif was GA-rich, similar to the pentamer motif determined for all crosslink sites with the exception that Cs were occasionally observed. This independent derivation of a binding sequence similar to the globally derived consensus indicates that SRSF4 binding to scaRNAs is specific. 
A.

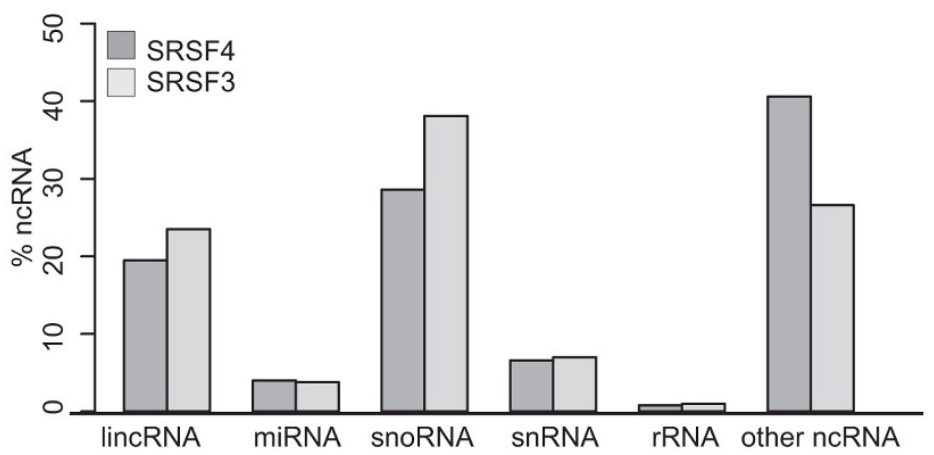

B.

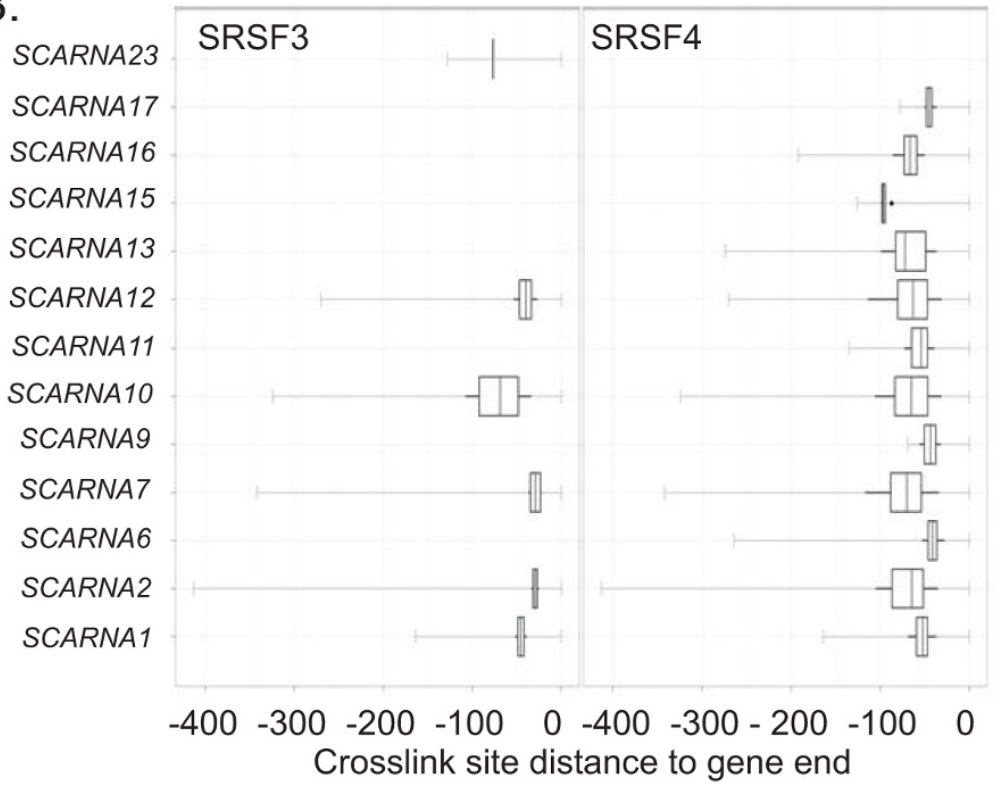

Figure 4 ncRNAs with SRSF3 and SRSF4 crosslink sites. (a) The distribution of crosslink sites within the ncRNA subclasses. (b) The position of the SRSF4 CLIP-tag clusters relative to the scaRNA 3' end. 'Other ncRNAs' are processed transcripts with no known ORF or function.

\section{SRSF3 and SRSF4 bind to intronless histone mRNAs} SRSF3 and SRSF4 binding sites were found in intronless protein-coding genes, likely reflecting SRSF3 and SRSF4 participation in regulatory events other than splicing. In particular, SRSF3 and SRSF4 CLIP-tag clusters were detected within histone genes: $73.8 \%$ of the mouse histone genes annotated in [25] had SRSF3 clusters and $47.7 \%$ had SRSF4 clusters (Figure 5a; Figure S4a in Additional file 1). This was also reflected in the enriched Gene Ontology (GO) terms where categories related to chromatin and nucleosome assembly were present (Table S4 in Additional file 1). The SRSF3 and SRSF4 CLIP-tag clusters were located at the boundary between ORF and 3' UTR and/or within the 3' UTR of histone mRNAs (Figure 5b). The CLIP-tag clusters were located just upstream of conserved stem-loops that occur 14 to
50 nucleotides downstream of the ORF (Figure 5a); these stem loops specify the sites of endonucleolytic cleavage of replication-dependent histone mRNAs and therefore define their 3' ends [26].

SRSF3 was previously shown to promote the export of histone $H 2 A$ reporter mRNAs via a 22-nucleotide transport element within the coding region of $H 2 A$ mRNAs, to which SRSF3 bound and recruited the mRNA export factor TAP $[27,28]$. In our study, however, most SRSF3 and SRSF4 CLIP-tag clusters in histone $H 2 A$ family mRNAs were found outside this 22-nucleotide transport element (Figure 5a, b; Figure S4a in Additional file 1). Furthermore, most SRSF3 and SRSF4 crosslink sites were present in mRNAs of histone families other than $H 2 A$, which do not contain the transport element (Additional file 2). Interestingly, SRSF3 and SRSF4 binding 


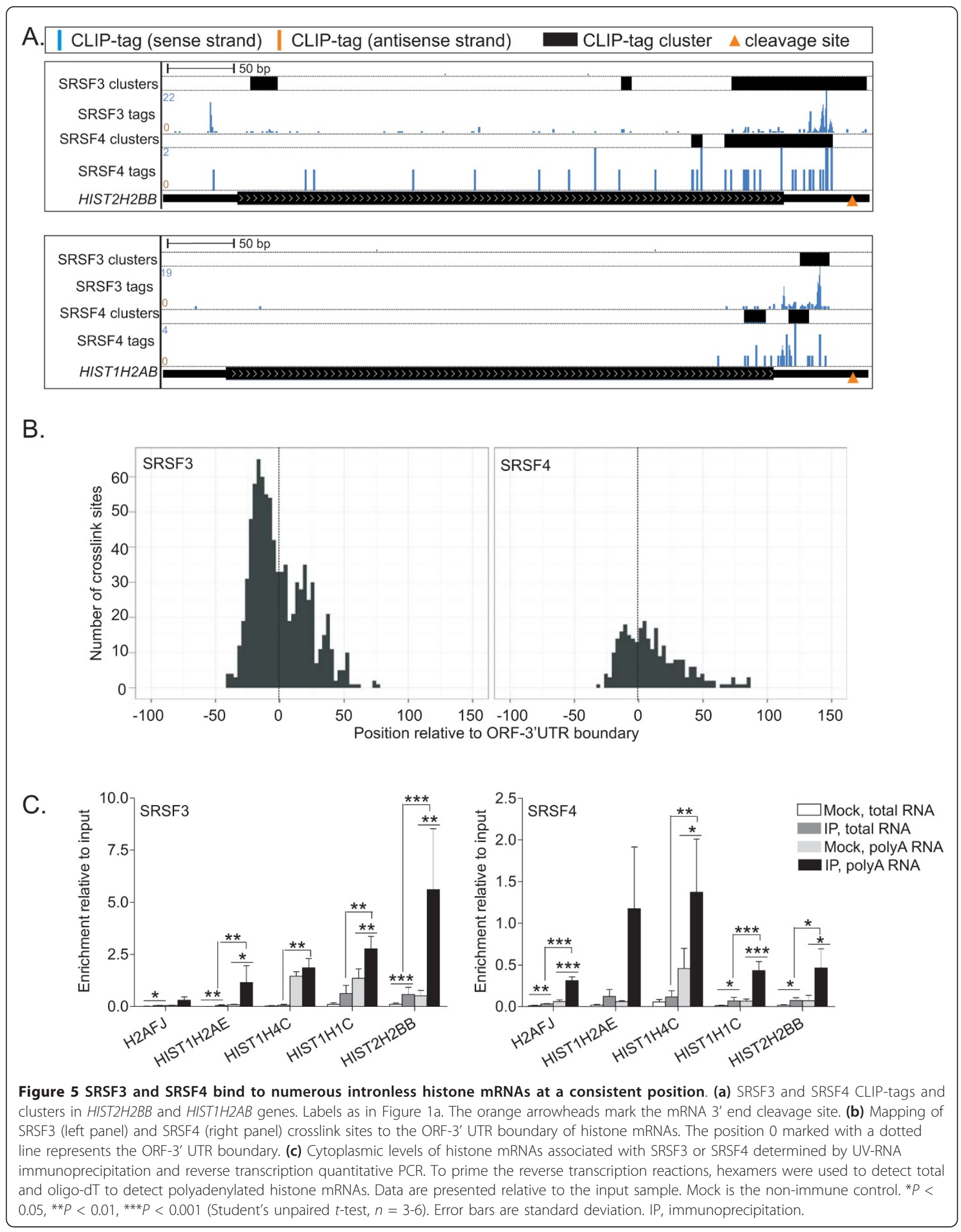


sites identified here are similar to those reported in another study that characterized export factor-binding sites in histone mRNAs [29].

SR proteins also promote polyadenylation in some contexts [30,31]. We found this intriguing in the context of the histone mRNA targets because several recent studies have shown that a significant pool of histone mRNAs undergo polyadenylation instead of 3' end cleavage [32-36]. To validate the association of SRSF3 and SRSF4 with histone mRNAs and to investigate polyadenylation, we adopted an RNA immunoprecipitation (RIP) assay from UV crosslinked cell extracts (UV-RIP); the immunoprecipitation was carried out from a cytoplasmic fraction in order to avoid contamination by genomic DNA that would later influence results obtained by reverse transcription quantitative PCR (RTqPCR) (Figure S4b in Additional file 1). Both total and polyadenylated histone mRNA levels were measured in the SRSF3 and SRSF4 immunoprecipitates, using either random hexamers or oligo-dT as reverse primers. Figure 5c shows that both SR proteins immunoprecipitated histone mRNAs significantly above mock immunoprecipitates, irrespective of which reverse primer was used. Compared to input, detection of histone mRNAs was more robust when oligo-dT reverse primers were used, suggesting that SRSF3 and SRSF4 preferentially bind polyadenylated histone mRNAs. The detection of SRSF3 and SRSF4 bound to polyadenylated histone mRNAs in the cytoplasmic fraction suggests that both SR proteins may be involved in histone mRNA 3' end formation, export, and/or translation.

\section{SRSF3 and SRSF4 make diverse contacts with exons and introns}

Because SR proteins are known to regulate pre-mRNA splicing, we wondered whether the crosslink sites were correlated with particular locations within introns and/ or exons. Data from in vitro studies suggest that SR proteins bind pre-mRNAs primarily within exons and thereby recruit spliceosomal components to adjacent 5 ' and 3' splice sites [37]. Therefore, crosslink sites were mapped to exon-intron and intron-exon boundaries. Variability in exon and intron length genome-wide leads to an apparent abundance of CLIP-tags close to the junctions (Figure S5a in Additional file 1). Therefore, we established a normalization factor derived from the length distribution of exons and introns to correct for these differences (Figure S5b in Additional file 1). Mapping of normalized crosslink sites showed exonic enrichment of SRSF3 and SRSF4 crosslink sites, which were most pronounced within 100 nucleotides of both $5^{\prime}$ and 3' splice sites (Figure 6a). Peaks of SRSF3 and SRSF4 binding approximately 70 nucleotides upstream of $5^{\prime}$ splice sites were more prominent than peaks observed downstream of 3' splice sites. Note that we did not map sequences falling onto exon-exon junctions, which explains the drop in crosslinking immediately upstream of 5' splice sites. Because SR proteins bind mRNA as well as pre-mRNA, it seems logical that exon sequences are overrepresented in the experimental data compared to intron sequences. However, similar patterns of enrichment in exons were observed when the pentamer motifs alone were considered (Figure 2; Figure S5c in Additional file 1), suggesting that the observed exon bias reflects the distribution of binding sequences within target RNAs. Interestingly, we noticed a peak of crosslink sites approximately 30 nucleotides upstream of 3' splice sites (Figure 6a). This corresponds to the approximate position of branch points in mammalian introns. However, the actual position of the branch point varies relative to the 3 ' splice site, with the longest observed distance of 400 nucleotides [38]. Therefore, crosslink sites were mapped to predicted mouse branch points [39]. This mapping indicated that SRSF3 and SRSF4 bind at or slightly downstream of the branch point nucleotide (Figure 6b). In conclusion, SRSF3 and SRSF4 preferentially contact exonic sequences, especially upstream of 5' splice sites; they also interact with branch points as suggested by two previous studies [7,40], consistent with the model that SR proteins regulate splicing by contacting pre-mRNA in different functional regions.

\section{SRSF3: a regulator of splicing factors}

The notion that different splicing factors might regulate transcripts with similar functions, creating an expression module regulated by splicing, has intrigued the field for decades. We therefore asked about the functional identity of SRSF3 and SRSF4 protein-coding targets. Similar to our previous findings by RIP-chip [13], GO analysis of the protein-coding genes with significant SRSF3 and SRSF4 crosslink sites revealed functions related to nucleic acid binding and RNA processing as the most enriched GO terms for both SRSF3 and SRSF4 (Table S4 in Additional file 1). SRSF3 binding sites were especially enriched within genes encoding components of RNP complexes, including splicing factors (Table S5 in Additional file 1). SRSF3 crosslink sites were found within the genes encoding other SR proteins, as well as in proteins of heterogeneous nuclear ribonucleoprotein complexes and components of the core splicing machinery. SRSF3 is known to strictly regulate its own expression through an inclusion of a premature termination codon (PTC)-containing cassette exon, which is referred to as a 'poison cassette exon' because it leads to transcript degradation by nonsense-mediated decay (NMD) $[13,41]$. Poison cassette exons occur in all SR protein family members and are ultraconserved among species $[42,43]$. The inclusion of the alternative cassette exon or 


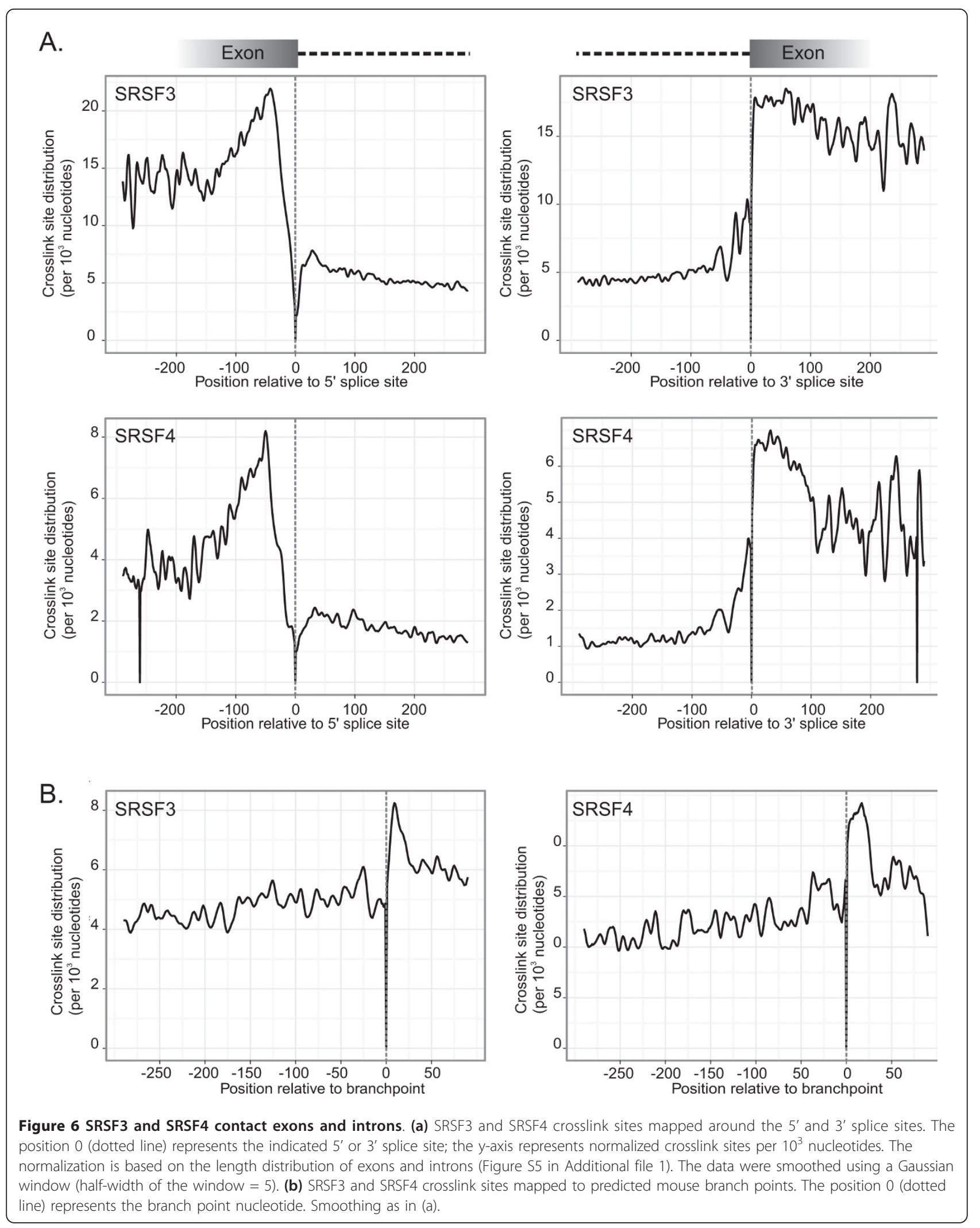


intron retention leads to the introduction of a PTC in the SR protein mRNA in every case. Indeed, SRSF3 and SRSF4 CLIP-tag clusters were detected in the SRSF3 and SRSF4 autoregulatory cassette exons, respectively (Figure 7a, top panel; Figure S6, bottom panel, in Additional file 1).

To date, it has been assumed that poison cassette exons are recognized by the gene's own protein product, in an auto-regulatory feedback loop (see above). Intriguingly, the SRSF3 CLIP-tag clusters were also found in the NMD-associated exons or introns of three heterologous SR protein-encoding genes, SRSF2, SRSF5 and SRSF7 (Figure 7a; Figure S6 in Additional file 1). In contrast, SRSF4 CLIP-tag clusters were found only in the poison cassette exon of its own pre-mRNA. We sought to validate the specificity of these interactions by UVRIP. SRSF3 specifically immunoprecipitated SRSF2, SRSF3, SRSF5 and SRSF7 (pre-)mRNAs, whereas SRSF4 only immunoprecipitated significant levels of its own (pre-)mRNA (Figure 7b). These data validate the specificity of SRSF3 interactions with heterologous transcripts encoding SR protein family members in the manner indicated by iCLIP; note that low recovery of some transcripts may be due to the short half-lives of the bound, PTC-containing messages.

The presence of SRSF3 CLIP-tag clusters in heterologous SR protein-encoding transcripts could indicate that SRSF3 either positively or negatively regulates poisoncassette exon usage. If so, we would predict that SRSF3 levels in cells should affect the alternative splicing and ultimately expression levels of the three target SR protein transcripts identified. To test this directly, minigenes including the genomic regions around SRSF3 CLIP-tag clusters were constructed for SRSF2, SRSF3, SRSF5 and SRSF7 (Figure 8a; Figure S7C in Additional file 1). Efficient SRSF3 or SRSF4 protein over-expression and knockdown was achieved by transfection of cDNA expression constructs and RNA interference, respectively (Figure S7a in Additional file 1). Under these conditions, the splicing patterns of the minigene-encoded transcripts were analyzed, using vector-specific primers for RT-PCR. Figure 8a shows that over-expression of SRSF3 led to a marked increase in poison cassette exon inclusion for both the SRSF3 and SRSF7 minigenes. Upon SRSF3 knockdown, this pattern was reversed (Figure S7b in Additional file 1). Similarly, SRSF3 over-expression led to alternative splicing changes for the SRSF2 and SRSF5 minigenes, leading to increased poison cassette usage and/or intron retention (Figure S7c in Additional file 1). Importantly, SRSF4 over-expression or knockdown did not detectably alter splicing patterns (Figure 8a; Figure S7b, c in Additional file 1).

The alternative splicing events regulated by SRSF3 documented above predict that the transcripts regulated by SRSF3 - namely SRSF3 itself as well as SRSF2, SRSF5 and SRSF7 - will undergo degradation through NMD when SRSF3 is over-expressed. To test this, the NMD pathway was inhibited by treating the cells with cycloheximide (CHX) [44]. The use of CHX as a tool also enabled us to investigate the alternative splicing outcome of endogenous transcripts. Figure 8b shows that $\mathrm{CHX}$ treatment leads to detection of the otherwise highly unstable endogenous poison cassette exon-containing SR protein transcripts that increase in abundance upon SRSF3 over-expression. Another prediction of these findings is that the steady-state levels of heterologous SR protein transcripts will depend on SRSF3 levels. Through measurement of target mRNA levels by RT-qPCR, we show that SRSF5 and SRSF7 mRNA levels decrease significantly in cells over-expressing SRSF3 (Figure 8c). Upon CHX treatment, mRNA levels recovered to those of the control (Figure S7d in Additional file 1). Taken together, the data indicate that SRSF3 specifically binds not only its own but other SR protein transcripts and the binding leads to alternative splicing changes that increase the occurrence of PTCs, which in turn target the expressed transcripts for degradation through the NMD pathway. Thus, SRSF3 regulates the expression of its own mRNA and the mRNAs encoding three other SR protein family members (Figure $8 d$ ). This cross-regulation by SRSF3 and the observation that many other RNA binding proteins may similarly be regulated by SRSF3 (Table S5 in Additional file 1) raises the possibility that SRSF3 is a master regulator of the transcriptome acting through a network of feedback mechanisms.

\section{Discussion}

Here we used iCLIP to investigate the RNA-binding landscape of two SR proteins, SRSF3 and SRSF4, in mouse cells. The value of this study is enhanced by the global comparison of RNA targets and binding sites for two members of this prominent family of RNA binding proteins with a variety of known roles in gene expression. Through detailed analysis of the transcripts and transcript regions bound by SRSF3 and SRSF4, we provide evidence for previously unknown functions of these highly conserved RNA binding proteins. Here we discuss our findings in the context of five major conclusions.

First, SRSF3 and SRSF4 exhibit largely non-overlapping binding sites and RNA targets, indicating that SRSF3 and SRSF4 regulate specific sets of genes through their interaction with different RNA sequences. This finding is consistent with the previous finding that SRSF3 and SRSF4 are present in non-overlapping mRNPs in vivo [13], although the previous study did not examine direct binding sites. The genome-wide CLIP data provided large numbers of binding sites, enabling 


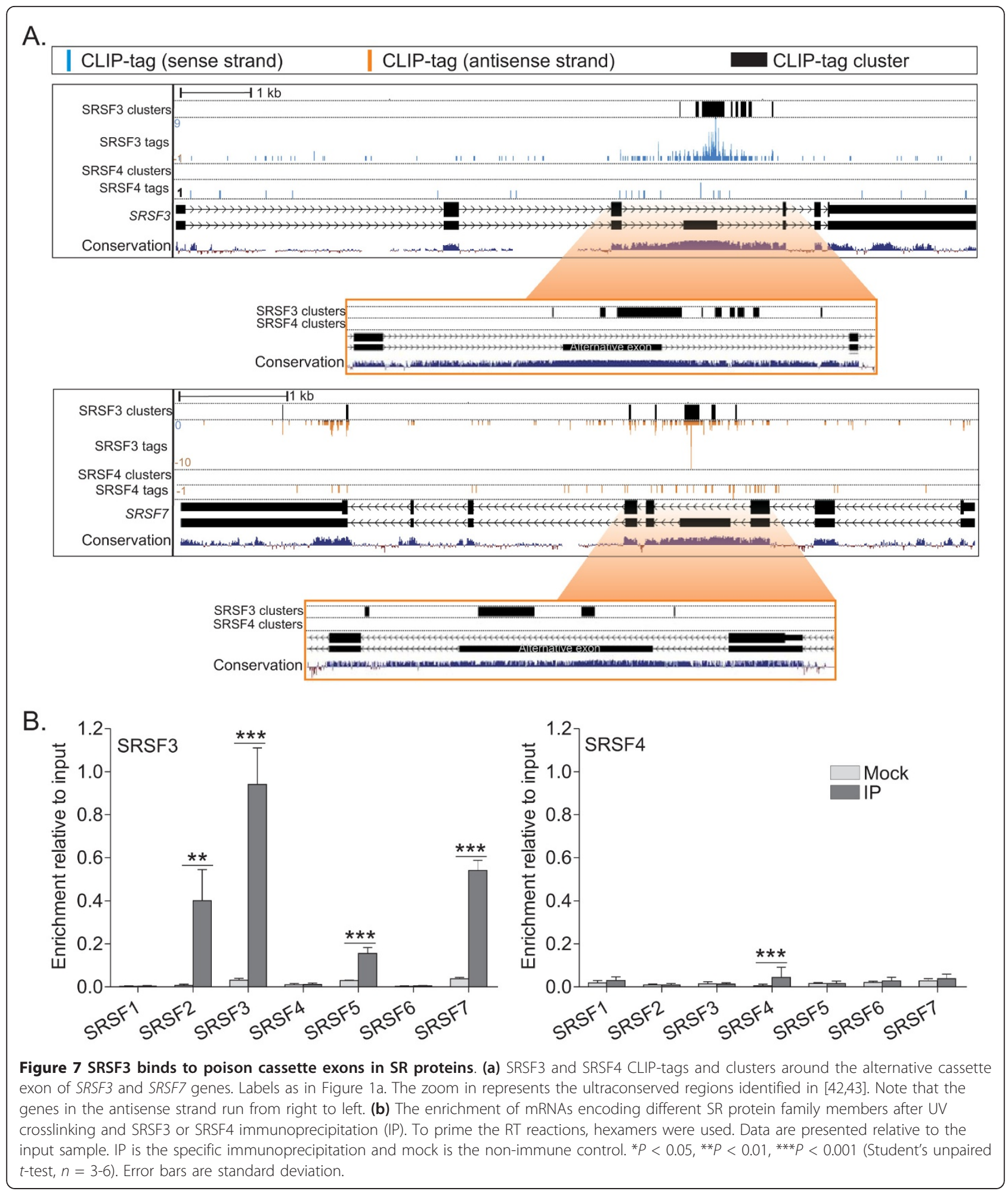

us to derive consensus in vivo binding sequences. We show that SRSF4 binds a consensus GA-rich sequence. The CU-rich SRSF3 in vivo consensus binding sequence is similar to that derived in vitro by SELEX $[18,19]$, indicating the validity of the use of SELEX to understand binding specificity alone. However, bioinformatic analysis of SELEX sequences does not permit the identification in vivo RNA targets because the shortness and 
A.

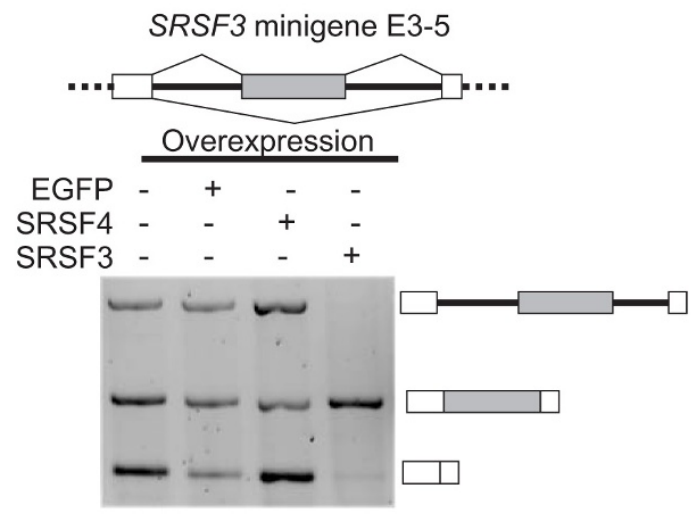

SRSF7 minigene E3-6

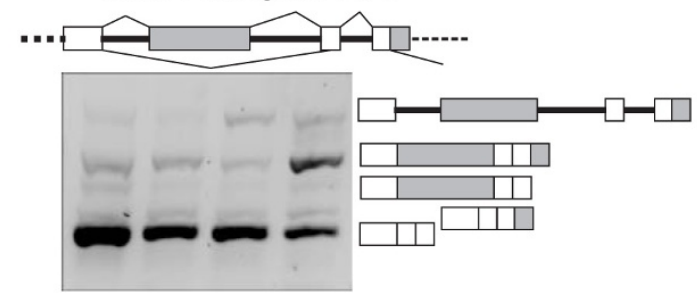

C.

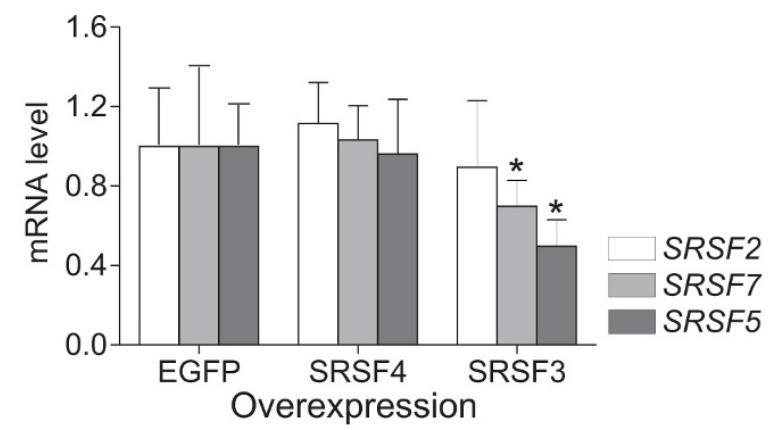

B.

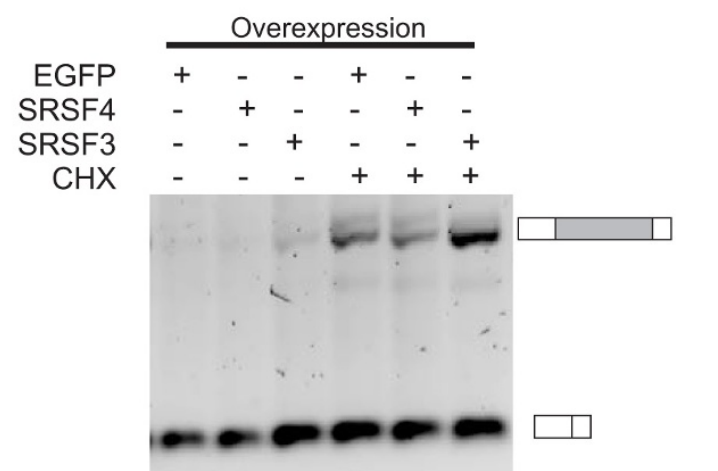

SRSF3 endogenous gene E3-5

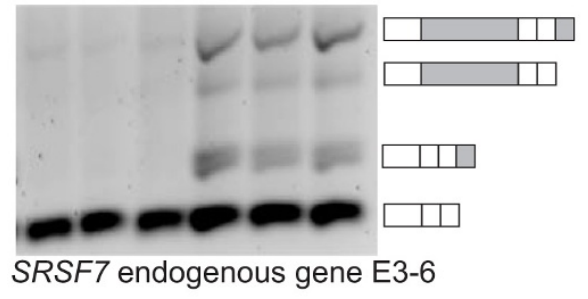

D.

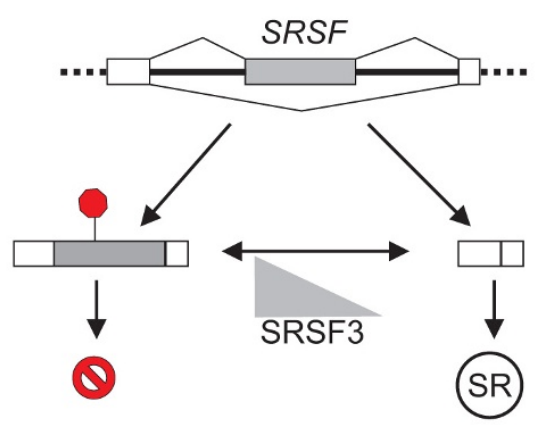

Figure 8 SRSF3 controls the level of SR proteins through splicing regulation. (a) The splicing products of SRSF3 and SRSF7 minigenes determined after 24-hour over-expression of SRSF3, SRSF4 or EGFP (control). The alternative exons are marked with light grey. (b) The splicing products of endogenous SRSF3 and SRSF7 after inhibition of NMD by a 3-hour treatment with cycloheximide (CHX). (c) The expression level of endogenous, mature SRSF2, SRSF5 and SRSF7 mRNAs upon EGFP, SRSF3 or SRSF4 overexpression (24 hours) as measured by RT-qPCR. * $P<0.05$ (one-way ANOVA). Error bars are standard deviation. ACTB was used as the reference gene. (d) Schematic showing how SRSF3 controls the levels of other SR protein family members through alternative splicing. The inclusion of a poison cassette exon harboring a premature termination codon (PTC, red stop sign) leads to RNA degradation through NMD.

degeneracy of consensus sequences leads to over-representation within the transcriptome [45]. The identification of in vivo targets, accomplished here by CLIP, allowed us to further investigate the identified RNA classes and RNA regions bound.

Second, an important class of RNAs bound by both SRSF3 and SRSF4 were ncRNAs. SRSF3 and SRSF4 crosslinked to multiple sites along the lincRNA $M A L A T 1$, which is enriched in nuclear speckles and interacts with a subset of SR proteins, including SRSF3
[22]. Other splicing factors localized in nuclear speckles, such as TDP-43 and SRSF1, also bind to MALAT1 $[12,21]$; it appears, therefore, that MALAT1 interaction is common among RNA-binding proteins in nuclear speckles. Furthermore, SRSF3 and SRSF4 interacted with $7 S K$, another ncRNA localized to speckles [23] but not with the paraspeckle ncRNA component NEAT1 [46]. One especially overrepresented group of short ncRNAs with SRSF3- and SRSF4-binding sites was snoRNAs. We show that a subset of snoRNAs, the 
scaRNAs, are prominent targets of SRSF4 with binding sites near their 3' ends. SR proteins are likely required for the splicing of all introns, including those containing snoRNAs. The position of the snoRNAs, including scaRNAs, within the host intron is critical for snoRNA processing, implying that the spliceosome is actively involved in the release of snoRNAs from the debranched intron [47]. It remains to be investigated whether SR proteins are required for snoRNA processing from host introns. An interesting possibility is that SR proteins interact within the snoRNA-derived short RNAs and coregulate alternative splicing [48]. Because the functions of many ncRNAs are currently poorly understood, it will be interesting to determine whether ncRNAs have functions as co-regulators of splicing.

Third, we provide evidence that SRSF3 and SRSF4 bind many intronless genes, further supporting their role as regulators of gene expression independent of pre-mRNA splicing [2]. Surprisingly, we show that SRSF3 and SRSF4 display clusters of binding sites at the ORF-3' UTR junction of the vast majority of histone mRNAs. This binding region was previously shown to be important for the export of histone H2A mRNAs in Xenopus oocytes [29]. UV-RIP experiments indicate that both SR proteins bind preferentially to polyadenylated histone mRNAs and that these mRNP complexes are detectable in the cytoplasm. Replication-dependent histone mRNAs are mainly processed by 3 ' end cleavage [26], and are exported from the nucleus via the stem loop binding protein (SLBP) [32]. It was known that histone mRNAs become polyadenylated when the 3' end cleavage machinery is compromised [26]. However, recent high-throughput sequence analyses of human and mouse mRNAs identified significant pools of polyadenylated mRNAs encoding all four core histones even when the 3 '-end processing machinery is functional $[35,36]$. Thus, expression of polyadenylated histone mRNAs appears to be physiologically important. If polyadenylated histone transcripts fail to bind SLBP, they may require SRSF3 and SRSF4 for export from the nucleus; both SRSF3 and SRSF4 shuttle to the cytoplasm and SRSF3 binds the mRNA export receptor TAP $[5,28]$. It will be of interest to determine how SRSF3 and SRSF4 regulate histone mRNA 3' end formation, export, or both.

Fourth, intron-containing protein-coding transcripts were a major class of SRSF3 and SRSF4 targets. SR proteins are thought to bind primarily to exonic splicing enhancers, where they influence recognition of adjacent 5 ' and 3' splice sites [49]. The pattern of binding to exon-intron junctions resembles that observed for SRSF1 [12]; abundant binding within the exon bodies dropped sharply towards exon-intron boundaries. These data agree with the current concept that SR proteins promote adjacent splice site recognition. However, our data show that neither SRSF3 nor SRSF4 binding is limited to exons; instead, a large number of binding sites are found in introns and 3' UTRs. Crosslinking to exonic regions should be overrepresented in CLIP, owing to the low abundance of pre-mRNA in living cells. It is remarkable, therefore, that $65 \%$ of SRSF3 and $52 \%$ of SRSF4 binding sites were located in introns. SRSF3 was shown to regulate splicing by binding to an intronic splicing enhancer [50], providing precedence for SRSF3 splicing regulatory function via intronic regulatory elements. Intriguingly, SRSF3 and SRSF4 binding was also detected at and around branch-point sequences within introns. In vitro studies suggested that SRSF1 is first recruited to an exonic splicing enhancer and the RS domain subsequently contacts the branch point $[7,40]$. The present study does not distinguish whether the RRM or the RS domain of SRSF3 and/or SRSF4 binds to the branchpoint. Although some correlation between binding to the branch point and to the downstream exon was observed, a strict requirement for binding to both downstream exonic splicing enhancer and the branch point was not detected. Therefore, SR proteins may have exon-independent functions as proposed previously [51].

Finally, we have discovered that SRSF3 cross-regulates the expression of other SR protein family members. Autoregulation, in which SRSF1 and SRSF3 modulate expression of their own messages via inclusion of a PTC-containing exon, was previously known $[41,52]$. This activity has been attributed to ultraconserved regions within alternative cassette exons and retained introns that introduce a PTC in mRNAs encoding SR proteins $[42,43]$. Here we identify SRSF3 binding sites in the mRNAs encoding three additional members of the SR protein family and show that short-term overexpression of SRSF3 led to robust effects on the splicing and expression of four SR protein family members (SRSF2, SRSF3, SRSF5, and SRSF7). This reveals an unexpected role of SRSF3 in cross-regulating expression of other SR protein family members (Figure 8d). SRSF3 binding sites were also abundant in mRNAs encoding other splicing factors and spliceosomal components. These data, together with the observation that SRSF3 binds numerous transcripts encoding RNA binding proteins, underscore the notion that the splicing machinery is under strict cellular control and indicate that SRSF3 is a key regulator of RNA metabolism.

\section{Conclusions}

Our global analysis of SRSF3 and SRSF4 shows that these SR proteins are multifunctional RNA binding proteins interacting with distinct classes of RNA. Initially identified as splicing factors, SRSF3 and SRSF4 regulate 
constitutive and alternative exons by binding to both exonic and intronic positions. SRSF3 in particular seems to function as a master regulator of splicing machinery expression through its activities in alternative splicing. However, SRSF3 and SRSF4 also interact with RNAs that are not processed by the spliceosome, suggesting a network of interactions that control cellular programs of gene expression. In addition to the numerous functions already assigned for SR proteins, interactions with different RNA classes, including ncRNAs, implies that more is yet to come.

\section{Materials and methods}

\section{Cell culture and iCLIP}

P19 cells were cultured as described [13]. For iCLIP, P19 SRSF3-BAC or P19 SRSF4-BAC cells [13] were irradiated with $100 \mathrm{~mJ} / \mathrm{cm}^{2}$ UV light. The iCLIP was performed as described [15]. Protein G Dynabeads coupled with goat anti-GFP antibody (a kind gift from D Drechsel, MPI-CBG, Dresden) were used for the immunopurification. The recovered RNA was reverse transcribed into cDNA. After size-purification and amplification the cDNA was subjected to highthroughput sequencing by Illumina Genome Analyser II (single-end 32-nucleotide reads). The adapter oligonucleotides, reverse transcription primers and primers for amplification were as described [15]. A more detailed description is in the Supplementary Methods in Additional file 1.

\section{Mapping of sequences to mouse genome and sequence analysis}

The sequences corresponding to an experiment were identified by a defined barcode and random barcodes were registered. The barcodes were removed before mapping to the mouse $\mathrm{mm} 9$ sequence assembly using Bowtie version 0.12.5. Two mismatches were allowed in the mapping, and only CLIP-tags mapping to unique positions were considered. For analysis of significant crosslink sites, the iCLIP positions were randomized. The randomization was done within co-transcribed regions as described [17]. Ensembl59 annotation based on the mouse $\mathrm{mm} 9$ genome assembly was used. The statistical approach used to identify significant crosslink sites and CLIP-tag clusters was as described [16,17].

Z-score analysis for enriched pentamers was performed essentially as described [17]. Pentamers were used because they are the longest motifs that could be statically derived from the data. Because we noticed that the inclusion of the actual crosslink nucleotide and the positions immediately surrounding it always resulted in a run of $U$ nucleotides as the most enriched motif (data not shown), we excluded the crosslink site from the analysis and thus avoided bias towards any nucleotide due to differences in crosslinking efficiency. The positions of the crosslinking nucleotide were extended by 30 nucleotides in both directions. Only one occurrence of a pentamer within the evaluated interval $[(-30,-10),(10,30)]$ relative to each cross-link was counted and each occurrence of a crosslink site was weighted by 1.0. Reference data were generated 100 times by random shuffling of iCLIP crosslink positions within corresponding genome segments (within same genes) and a Z-score was calculated relative to the randomized genomic positions. The top five pentamers were used to calculate the binding consensus motif [53].

\section{Mapping of crosslink sites to exon-intron junctions and branch points}

Crosslink sites located within a maximum 600-nucleotide window $[-300,+300]$ around exon-intron boundaries were mapped to these regions. Each occurrence of a crosslink site was assigned to the closest exon-intron boundary, counted as 1.0 and normalized by number of junctions spanning the crosslink position. In the case of exon-intron junctions, only the last half of the exon and first half of the intron were used to obtain the distribution of exons and introns spanning each position relative to the boundary. Similarly, the last half of introns and the first half of exons were used for intron-exon junctions. Junctions where exons were shorter than 60 nucleotides or introns shorter than 200 nucleotides were ignored ( $<15 \%$ of all possible junctions). For branch point RNA maps, we used computationally predicted branch points from Corvelo et al. [39]. Only the best, non-negative SVM scored branch point that resided in the last half of intron was used. In case of ties, we used the branch point closest to the intron-exon junction. For normalization, only the last half of the intron was used, and the branch point was at position zero. We ignored introns shorter than 240 nucleotides and introns where the branch point was closer than 20 nucleotides to the intron-exon junction.

\section{Minigene analysis}

For the knockdown of SRSF3 or SRSF4, esiRNA sequences described previously were used [13]. For the overexpression, human SRSF3 and SRSF4 cDNA were cloned into pYFP-N2 and pEGFP-N3 vectors (Clontech), respectively. To construct the minigenes, the region of interest was PCR amplified from P19 genomic DNA and cloned into pcDNA3 vector (Invitrogen). The plasmids carrying the minigenes were co-transfected together with either SRSF3 or SRSF4 esiRNA or overexpression construct into P19 cells using Lipofectamine 2000 (Invitrogen). Empty vectors or esiRNA targeting EGFP were used as controls. Twentyfour hours post-transfection, total RNA was isolated using acid phenol-chloroform extraction (Ambion). After 
DNaseI treatment the RNA was reverse transcribed with Superscript III (Invitrogen). The splicing patterns of minigenes were analyzed using vector-specific PCR primers. To abrogate the nonsense-mediated decay pathway, P19 wt cells were treated with $300 \mu \mathrm{g} / \mathrm{ml}$ cycloheximide for 3 hours. Untreated samples were processed in parallel. Total RNA was extracted and the samples treated as described above. Gene-specific primers spanning the exons flanking the PTC-containing cassette exon were used. Total expression of SRSF2, SRSF5 and SRSF7 was determined by RTqPCR using primers spanning an exon-exon junction. $A C T B$ was used as a reference gene to normalize for cDNA synthesis efficiency. Primer sequences are available upon request.

\section{UV crosslinking and RNA immunoprecipitation}

UV-crosslinked cells (see above) were harvested and the cytoplasmic fraction was separated. The total cell pellet was suspended in NET-2 buffer $(50 \mathrm{mM}$ Tris- $\mathrm{HCl} \mathrm{pH}$ 7.4, $150 \mathrm{mM} \mathrm{NaCl} ; 0.05 \%$ (v/v) Nonidet P-40) for western blot analysis or Trizol (Invitrogen) for RNA isolation. For the fractionation, the cell pellet was suspended in hypotonic buffer $(10 \mathrm{mM}$ Hepes, $\mathrm{pH} 7.4 ; 10 \mathrm{mM}$ $\mathrm{NaCl} ; 3 \mathrm{mM} \mathrm{MgCl}{ }_{2} ; 0.3 \%$ (v/v) Nonidet P-40, RNaseOUT; complete protein inhibitor cocktail), the nuclear pellet was collected by centrifugation, and the supernatant was collected as the cytoplasmic faction. The cytoplasmic fraction was used as such or RNA was extracted as above. Input, mock and immunoprecipitation samples were independently reverse primed with either oligo-dT or hexamer primers. Primers specific for individual histone mRNAs were used for qPCR amplification; POFUT1 was used as a reference gene for SRSF3 and $D T M Y K$ for SRSF4 to normalize for cDNA synthesis efficiency. Oligo-dT and hexamer samples were normalized independently of each other to their respective input. The primer sequences are available upon request.

\section{Database accession}

The sequencing data have been submitted to the Array Express database [54], accession number E-MTAB-747.

\section{Additional material}

Additional file 1: Supplementary Information. Supplementary

Materials and methods, References, Figures S1 to S7 and Tables S1 and S3 to S5.

Additional file 2: Supplementary Table S2. Genes with significant SRSF3 or SRSF4 crosslink sites.

\section{Abbreviations}

BAC: bacterial artificial chromosome; CHX: cycloheximide; CLIP: crosslinking and immunoprecipitation; EGFP: enhanced green fluorescent protein; GO: Gene Ontology; iCLIP: individual nucleotide-resolution UV cross-linking and immunoprecipitation; lincRNA: long non-coding RNA; mRNP: mitochondrial ribonucleoprotein ; ncRNA: non-coding RNA; NMD: nonsense-mediated decay; ORF: open reading frame; PTC: premature termination codon; RIP: RNA immunoprecipitation; RNP: ribonucleoprotein; RRM: RNA recognition motif; RT-(q)PCR: reverse transcription (quantitative) polymerase chain reaction; scaRNA: small Cajal body-specific RNA; SELEX: systemic evolution of ligands by exponential enrichment; SLBP: stem loop binding protein; snoRNA: small nucleolar RNA; UTR: untranslated region; UV: ultraviolet.

\section{Acknowledgements}

We thank members of the Neugebauer lab, Michael Hiller, and Tim Mercer for helpful discussions and comments on the manuscript. The financial support was from Sigrid Juselius foundation (to M-LÄ), Helsingin Sanomain Foundation (to M-LÄ), EMBO long-term fellowship (to MMM), Slovenian Research Agency (Z7-3665 to TC), the Max Planck Society (to KMN), the Deutsche Forschungsgemeinschaft (NE909/3-1 to KMN).

\section{Author details}

${ }^{1}$ Max Planck Institute of Cell Biology and Genetics, Pfotenhauerstr. 108, Dresden, 01307, Germany. ${ }^{2}$ Laboratory of Molecular Biology, Medical Research Council, Hills Road, Cambridge, CB2 0QH, UK. ${ }^{3}$ Faculty of Computer and Information Science, University of Ljubljana, Trzaska cesta 25, Ljubljana, SI-1001, Slovenia. ${ }^{4}$ Current address: Australian Regenerative Medicine Institute, Monash University, Wellington Road, Clayton, Melbourne, VIC-3800, Australia.

\section{Authors' contributions}

$M-L \ddot{A}, M M M$ and KMN designed the experiments; M-LÄ and MMM performed the experiments; $M-L \ddot{A}, H B, I H, J U, T C$ and $C G$ analyzed the data; $M-L \ddot{A}, M M M$ and KMN wrote the manuscript. The final manuscript has been read and approved by all the authors.

\section{Competing interests}

The authors declare that they have no competing interests.

Received: 26 January 2012 Revised: 20 March 2012

Accepted: 21 March 2012 Published: 21 March 2012

\section{References}

1. Letunic I, Doerks T, Bork P: SMART 6: recent updates and new developments. Nucleic Acids Res 2009, 37:D229-D232.

2. Zhong $X-Y$, Wang $P$, Han J, Rosenfeld MG, Fu X-D: SR proteins in vertical integration of gene expression from transcription to RNA processing to translation. Mol Cell 2009, 35:1-10.

3. Shepard P, Hertel K: The SR protein family. Genome Biol 2009, 10:242.

4. Lin S, Fu XD: SR proteins and related factors in alternative splicing. Adv Exp Med Biol 2007, 623:107-122.

5. Sapra AK, Änkö M-L, Grishina I, Lorenz M, Pabis M, Poser I, Rollins J, Weiland E-M, Neugebauer KM: SR protein family members display diverse activities in the formation of nascent and mature mRNPs in vivo. $\mathrm{Mol}$ Cell 2009, 34:179-190.

6. Caceres JF, Screaton GR, Krainer AR: A specific subset of SR proteins shuttles continuously between the nucleus and the cytoplasm. Genes Dev 1998, 12:55-66.

7. Shen H, Kan JLC, Green MR: Arginine-serine-rich domains bound at splicing enhancers contact the branchpoint to promote prespliceosome assembly. Mol Cell 2004, 13:367-376.

8. Mayeda A, Screaton GR, Chandler SD, Fu X-D, Krainer AR: Substrate specificities of SR proteins in constitutive splicing are determined by their RNA recognition motifs and composite pre-mRNA exonic elements. Mol Cell Biol 1999, 19:1853-1863.

9. Caceres JF, Misteli T, Screaton GR, Spector DL, Krainer AR: Role of the modular domains of SR proteins in subnuclear localization and alternative splicing specificity. J Cell Biol 1997, 138:225-238.

10. Ray D, Kazan H, Chan ET, Castillo LP, Chaudhry S, Talukder S, Blencowe BJ, Morris Q, Hughes TR: Rapid and systematic analysis of the RNA recognition specificities of RNA-binding proteins. Nat Biotechnol 2009, 27:667-670.

11. Blencowe BJ: Alternative splicing: new insights from global analyses. Cell 2006, 126:37-47. 
12. Sanford JR, Wang X, Mort M, VanDuyn N, Cooper DN, Mooney SD, Edenberg HJ, Liu Y: Splicing factor SFRS1 recognizes a functionally diverse landscape of RNA transcripts. Genome Res 2009, 19:381-394.

13. Änkö M-L, Morales L, Henry I, Beyer A, Neugebauer KM: Global analysis reveals SRp20- and SRp75-specific mRNPs in cycling and neural cells. Nat Struct Mol Biol 2010, 17:962-970.

14. Wang Z, Tollervey J, Briese M, Turner D, Ule J: CLIP: Construction of CDNA libraries for high-throughput sequencing from RNAs cross-linked to proteins in vivo. Methods 2009, 48:287-293.

15. Konig J, Zarnack K, Rot G, Curk T, Kayikci M, Zupan B, Turner DJ, Luscombe NM, Ule J: iCLIP reveals the function of hnRNP particles in splicing at individual nucleotide resolution. Nat Struct Mol Biol 2010, 17:909-915.

16. Yeo GW, Coufal NG, Liang TY, Peng GE, Fu X-D, Gage FH: An RNA code for the FOX2 splicing regulator revealed by mapping RNA-protein interactions in stem cells. Nat Struct Mol Biol 2009, 16:130-137.

17. Wang Z, Kayikci M, Briese M, Zarnack K, Luscombe NM, Rot G, Zupan B, Curk T, Ule J: iCLIP predicts the dual splicing effects of TIA-RNA interactions. PLOS Biol 2010, 8:e1000530

18. Cavaloc Y, Bourgeois CF, Kister L, Stevenin J: The splicing factors $9 G 8$ and SRp20 transactivate splicing through different and specific enhancers. RNA 1999, 5:468-483.

19. Schaal TD, Maniatis T: Selection and characterization of pre-mRNA splicing enhancers: identification of novel SR protein-specific enhancer sequences. Mol Cell Biol 1999, 19:1705-1719.

20. Zheng ZM, He PJ, Baker CC: Structural, functional, and protein binding analyses of bovine papillomavirus type 1 exonic splicing enhancers. $J$ Virol 1997, 71:9096-9107.

21. Tollervey JR, Curk T, Rogelj B, Briese M, Cereda M, Kayikci M, König J, Hortobágyi T, Nishimura AL, Zupunski V, Patani R, Chandran S, Rot G, Zupan B, Shaw CE, Ule J: Characterizing the RNA targets and positiondependent splicing regulation by TDP-43. Nat Neurosci 2011, 14:452-458.

22. Tripathi V, Ellis JD, Shen Z, Song DY, Pan Q, Watt AT, Freier SM, Bennett CF, Sharma A, Bubulya PA, Blencowe BJ, Prasanth SG, Prasanth KV: The nuclearretained noncoding RNA MALAT1 regulates alternative splicing by modulating SR splicing factor phosphorylation. Mol Cell 2010, 29:925-938.

23. Prasanth KV, Camiolo M, Chan G, Tripathi V, Denis L, Nakamura T, Hubner MR, Spector DL: Nuclear organization and dynamics of 7SK RNA in regulating gene expression. Mol Biol Cell 2010, 21:4184-4196.

24. Kiss T: Small nucleolar RNA-guided post-transcriptional modification of cellular RNAs. EMBO J 2001, 20:3617-3622.

25. Marzluff WF, Gongidi P, Woods KR, Jin J, Maltais LJ: The human and mouse replication-dependent histone genes. Genomics 2002, 80:487-498.

26. Marzluff WF, Wagner EJ, Duronio RJ: Metabolism and regulation of canonical histone mRNAs: life without a poly(A) tail. Nat Rev Genet 2008, 9:843-854.

27. Huang Y, Steitz JA: Splicing factors SRp20 and $9 \mathrm{G} 8$ promote the nucleocytoplasmic export of mRNA. Mol Cell 2001, 7:899-905.

28. Huang Y, Gattoni R, Stévenin J, Steitz JA: SR splicing factors serve as adapter proteins for TAP-dependent mRNA export. Mol Cell 2003, 11:837-843.

29. Erkmann JA, Sanchez R, Treichel N, Marzluff WF, Kutay U: Nuclear export of metazoan replication-dependent histone mRNAs is dependent on RNA length and is mediated by TAP. RNA 2005, 11:45-58.

30. Hudson SW, McNally MT: Juxtaposition of two distant, serine-arginine-rich protein-binding elements is required for optimal polyadenylation in rous sarcoma virus. J Virol 2011, 85:11351-11360.

31. Lou H, Neugebauer KM, Gagel RF, Berget SM: Regulation of alternative polyadenylation by U1 snRNPs and SRp20. Mol Cell Biol 1998, 18:4977-4985.

32. Sullivan KD, Mullen TE, Marzluff WF, Wagner EJ: Knockdown of SLBP results in nuclear retention of histone mRNA. RNA 2009, 15:459-472.

33. Godfrey AC, Kupsco JM, Burch BD, Zimmerman RM, Dominski Z, Marzluff WF, Duronio RJ: U7 snRNA mutations in Drosophila block histone pre-mRNA processing and disrupt oogenesis. RNA 2006, 12:396-409.

34. Wagner EJ, Burch BD, Godfrey AC, Salzler HR, Duronio RJ, Marzluff WF: A genome-wide RNA interference screen reveals that variant histones are necessary for replication-dependent histone pre-mRNA processing. $\mathrm{Mol}$ Cell 2007, 28:692-699.
35. Shepard PJ, Choi E-A, Lu J, Flanagan LA, Hertel KJ, Shi Y: Complex and dynamic landscape of RNA polyadenylation revealed by PAS-Seq. RNA 2011, 17:761-772.

36. Yang L, Duff M, Graveley B, Carmichael G, Chen L-L: Genomewide characterization of non-polyadenylated RNAs. Genome Biol 2011, 12:R16.

37. Graveley BR, Hertel K, Maniatis T: SR proteins are 'locators' of the RNA splicing machinery. Curr Biol 1999, 9:R6-R7.

38. Gooding C, Clark F, Wollerton M, Grellscheid S-N, Groom H, Smith C: A class of human exons with predicted distant branch points revealed by analysis of AG dinucleotide exclusion zones. Genome Biol 2006, 7:R1.

39. Corvelo A, Hallegger M, Smith CWJ, Eyras E: Genome-wide association between branch point properties and alternative splicing. PLoS Comput Biol 2010, 6:e1001016.

40. Shen H, Green MR: RS domains contact splicing signals and promote splicing by a commonmechanism in yeast through humans. Genes Dev 2006, 20:1755-1765.

41. Jumaa H, Nielsen PJ: The splicing factor SRp20 modifies splicing of its own mRNA and ASF/SF2 antagonizes this regulation. EMBO J 1997, 16:5077-5085.

42. Ni JZ, Grate L, Donohue JP, Preston C, Nobida N, O'Brien G, Shiue L, Clark TA, Blume JE, Ares M Jr: Ultraconserved elements are associated with homeostatic control of splicing regulators by alternative splicing and nonsense-mediated decay. Genes Dev 2007, 21:708-718.

43. Lareau LF, Inada M, Green RE, Wengrod JC, Brenner SE: Unproductive splicing of SR genes associated with highly conserved and ultraconserved DNA elements. Nature 2007, 446:926-929.

44. Saltzman AL, Pan Q, Blencowe BJ: Regulation of alternative splicing by the core spliceosomal machinery. Genes Dev 2011, 25:373-384.

45. Wang J, Smith PJ, Krainer AR, Zhang MQ: Distribution of SR protein exonic splicing enhancer motifs in human protein-coding genes. Nucleic Acids Res 2005, 33:5053-5062.

46. Bond CS, Fox AH: Paraspeckles: nuclear bodies built on long noncoding RNA. J Cell Biol 2009, 186:637-644.

47. Hirose T, Steitz JA: Position within the host intron is critical for efficient processing of box C/D snoRNAs in mammalian cells. Proc Natl Acad Sci USA 2001, 98:12914-12919.

48. Khanna A, Stamm S: Regulation of alternative splicing by short noncoding nuclear RNAs. RNA Biol 2011, 7:480-485.

49. Blencowe BJ: Exonic splicing enhancers: mechanism of action, diversity and role in human genetic diseases. Trends Biochem Sci 2000, 25:106-110.

50. Lou H, Neugebauer KM, Gagel RF, Berget SM: Regulation of alternative polyadenylation by U1 snRNPs and SRp20. Mol Cell Biol 1998, 18:4977-4985.

51. Hertel KJ, Maniatis T: Serine-arginine (SR)-rich splicing factors have an exon-independent function in pre-mRNA splicing. Proc Natl Acad Sci USA 1999, 96:2651-2655.

52. Sun S, Zhang Z, Sinha R, Karni R, Krainer AR: SF2/ASF autoregulation involves multiple layers of post-transcriptional and translational control. Nat Struct Mol Biol 2010, 17:306-312.

53. Crooks GE, Hon G, Chandonia J-M, Brenner SE: WebLogo: a sequence logo generator. Genome Res 2004, 14:1188-1190.

54. Array Express.. [http://www.ebi.ac.uk/arrayexpress/].

doi:10.1186/gb-2012-13-3-r17

Cite this article as: Änkö et al:: The RNA-binding landscapes of two SR proteins reveal unique functions and binding to diverse RNA classes. Genome Biology 2012 13:R17. 